\title{
O acolhimento e o Núcleo de Apoio à Saúde da Família no município do Rio de Janeiro: fragmentos, perspectivas e reflexões
}

\author{
"User embracement" and the Family Health Support Team in the city of Rio de Janeiro: fragments, perspectives and reflections \\ El acogimiento y el Núcleo de Apoyo a la Salud de la Familia en el municipio de Rio de Janeiro: fragmentos, \\ perspectivas y reflexiones.
}

Tarciso Feijó da Silva. Universidade do Estado do Rio de Janeiro (UERJ). Rio de Janeiro, RJ, Brasil. tarcisofeijo@yahoo.com.br (Autor correspondente) Valéria Ferreira Romano. Universidade Federal do Rio de Janeiro (UFRJ). Rio de Janeiro, RJ, Brasil. valromano@uol.com.br

\section{Resumo}

Objetivos: identificar a percepção de diferentes categorias profissionais da área da saúde sobre 0 acolhimento e sobre 0 trabalho do Núcleo de Apoio à Saúde da Família (NASF) e analisar o modo como esses mesmos profissionais realizam o acolhimento. Métodos: abordagem qualitativa com utilização de técnicas de observação sistemática e entrevistas semiestruturadas com profissionais de duas Unidades Básicas de Saúde do município do Rio de Janeiro. Os dados foram analisados considerando as referências de Bardin, a partir da Análise de Conteúdo, onde as inferências são valorizadas. Resultados: foram observadas fragilidades importantes relacionadas ao acolhimento e um NASF pouco envolvido com o desenvolvimento de competências da equipe. Conclusão: 0 acolhimento, enquanto dispositivo que contribui para garantia do acesso, ampliação da clínica e aumento da resolutividade, foi visto como essencial para produção do cuidado, e o NASF,na medida em que qualifica as ações,como potente ferramenta de gestão.

\section{Abstract}

Objectives: to identify the perceptions of different categories of health professionals about "user embracement" and about the work done by the Family Health Support Team (NASF) and to analyze the way in which "user embracement" is carried out by these professionals. Methods: qualitative approach using systematic observation techniques and semi structured interviews with professionals from two primary health care units from the city of Rio de Janeiro. The data were analyzed based on Bardin's framework, where Content Analyses was used to make inferences. Results: important weaknesses were seen in the implementation of "user embracement", and the Family Health Support Team (NASF) showed little engagement in the development of team skills. Conclusions: "user embracement" contributed to ensuring access, expanding clinical outreach and increasing clinical efficiency and was seen as essential to the production of care. The NASF helped to qualify clinical practice and was seen as a powerful management tool.

\section{Resumen}

Objetivos: identificar la percepción de las diferentes categorías de profesionales de la salud acerca del acogimiento y el trabajo en el Núcleo de Apoyo a la Salud de la Familia (NASF) y analizar la forma de llevar a cabo el acogimiento por los mismos profesionales.Métodos: estudio cualitativo utilizando técnicas de observación sistemática y entrevistas semi-estructuradas con profesionales en dos Unidades Básicas de Salud en la Ciudad de Rio de Janeiro. Los datos obtenidos fueron analizados teniendo en cuenta las referencias de Bardin, a partir del Análisis de Contenido, donde se valoran las inferencias. Resultados: se observaron debilidades significativas relacionadas con el acogimiento, además del encuentro de un NASF poco involucrado con el desenvolvimiento de las competencias del equipo. Conclusión: el acogimiento, mientras que el dispositivo que contribuya a garantizar el acceso, la expansión de la clínica y el aumento de la eficiencia, fue visto como esencial para la producción de la atención, y el NASF ayudó a calificar las acciones, siendo visto como una potente herramienta de gestión.
Palavras-chave:

Administração de Serviços de Saúde

Acolhimento

Estratégia Saúde da Família

Keywords:

Health Services Administration User Embracement Family Health Strategy

Palabras clave: Administración de los Servicios de Salud Acogimiento Estrategia de Salud Familiar 


\section{Introdução}

A organização do processo de trabalho das equipes da Estratégia Saúde da Família no município do Rio de Janeiro, apesar da existência de um discurso teórico contrário, prevalece, em sua essência e produção, funcionando a partir de uma intensa divisão do trabalho, seja devido ao paradigma biomédico, matriz da formação em saúde, seja porque pouco exercita um trabalho integrado. Alguns autores apontam como fator de divisão do trabalho na Saúde da Família a convivência entre o modelo da integralidade e os programas verticais do Ministério da Saúde. ${ }^{1}$ De qualquer maneira, um processo produtivo fragmentado e isolado tem sido a tônica, tirando força de escuta sobre as demandas de atendimento, o que acaba por construir espaços de conflito entre as propostas de atenção e a vontade dos usuários. O grande agravante é que esse cenário pode acarretar na "alienação do trabalhador diante do produto final", ${ }^{2}$ na manutenção da execução de tarefas repetitivas e desconectadas e na crença de que não há outra forma de se trabalhar em saúde. Como consequência, usuários e profissionais saem desta relação insatisfeitos.

Assim, a estrutura tradicional dos serviços de saúde reforça o isolamento profissional e reproduz uma fragmentação do processo de trabalho, mantendo a inquestionável hegemonia do paradigma biomédico, como citado, e a tendência atual do profissional em se responsabilizar pelos procedimentos característicos específicos do seu núcleo de conhecimento, restringindo o trabalhador de pensar e agir sobre a história de vida das pessoas. ${ }^{3}$ Dessa maneira, as açôes de saúde tendem a ser pré-estabelecidas na maioria das equipes; além disso, a coordenação e supervisão destas acontecem de forma hierárquica entre gestores e trabalhadores, sem a participação efetiva dos usuários ou consistente discussão prévia de temas. ${ }^{1}$

Quando consideramos a perspectiva do trabalho em equipe na Estratégia Saúde da Família em relação à tomada de decisão para diagnósticos ou intervençóes terapêuticas, inferimos que, de maneira geral, estas tendem a ser solitárias e raramente compartilhadas com a equipe. A formação, a gestão e o planejamento em saúde necessitam rever e incorporar saberes e técnicas que valorizem a inter-relação entre equipes e população, ${ }^{4}$ possibilitando uma "reordenação do trabalho em saúde por meio do vínculo terapêutico entre equipe e usuários, estimulando a produção progressiva de um novo padrão de responsabilidade pela co-produção de saúde". ${ }^{3}$

Merhy sugere a utilização de instrumentos gerenciais que propiciem a "auto-análise" e a "auto-gestão" dos trabalhadores de saúde para a reorientação da atenção básica pela Estratégia Saúde da Família. ${ }^{5}$ Para além de questionar o que ocorre nos serviços, o autor compreende que se deve desenvolver a capacidade de "criar interrogaçóes" sobre o que está sendo vivenciado, abrindo com isso possibilidades para o trabalhador coletivo inventar modos novos e singulares de realizar o trabalho em saúde em situaçóes concretas. ${ }^{6}$ Nesse contexto, e na tentativa de recolher fragmentos, o acolhimento é compreendido não só como uma forma humanizada, respeitosa e comprometida que deve estar presente na relação profissional-usuário, mas como um recurso para garantia do acesso aos serviços disponíveis pela Atenção Primária. ${ }^{7}$ Deve ser visto, como elemento primordial do processo de trabalho em saúde, enquanto concepçáo centrada nas tecnologias leves, nas relaçóes e no cuidado ampliado. Divergindo da organização atual dos serviços de saúde, centrado nas tecnologias duras, intrinsecamente dependentes de equipamentos e nas leve-duras, caracterizadas pelo domínio de um núcleo específico de conhecimentos, como a consulta médica e de enfermagem, o acolhimento produz possibilidades.

Se a partir do acolhimento poderão surgir açôes de responsabilização, resolução e construção da autonomia do usuário, o valorizamos neste artigo principalmente devido à sua implicação direta na forma de uso dos serviços ofertados.

Considerando o exposto, este artigo tem o objetivo de abordar a temática acolhimento considerando duas vertentes: identificar a percepçáo de diferentes categorias de profissionais de saúde sobre o acolhimento e sobre o trabalho no Núcleo de Apoio à Saúde da Família (NASF) e analisar o modo de realização do acolhimento pelos mesmos profissionais.

\section{Metodologia}

Este artigo apresenta o resultado de uma pesquisa de campo, de base qualitativa, que realizou um estudo descritivo e exploratório em duas Unidades Básicas de Saúde (UBS) no Município do Rio de Janeiro, sendo as mesmas denominadas de acordo com a Carteira de Serviços do Município do Rio de Janeiro: Uma Clínica da Família (CF) e um Centro Municipal de Saúde $(\mathrm{CMS})^{8}$. O projeto foi aprovado pelo Comitê de Ética em Pesquisa da Escola Nacional de Saúde Pública Sérgio Arouca (ENSP/FIOCRUZ) sob o no 182.519 , além de ter recebido anuência institucional da Secretaria Municipal de Saúde do Rio de Janeiro. O estudo foi realizado entre os meses de julho de 2012 a agosto de 2013. 
Os sujeitos da pesquisa foram 11 profissionais de cada UBS, um de cada das seguintes categorias: médico, enfermeiro, dentista, técnico de enfermagem, técnico de higiene dental, agente de vigilância em saúde, agente comunitário de saúde, auxiliar de portaria, auxiliar de serviços gerais, uma profissional nutricionista do Núcleo de Apoio à Saúde da Família (NASF) e o gerente de cada uma das UBS. Alguns critérios foram utilizados para a escolha quando havia mais de um profissional daquela categoria na UBS: ter especialização na área da Atenção Primária, quando profissional médico, enfermeiro, dentista e NASF, e outros cursos relacionados à Atenção Primária, quando profissionais de nível médio. Na ausência destes critérios, foram entrevistados os profissionais com maior tempo de atuação na unidade.

A priori foi realizado um estudo bibliográfico ${ }^{9}$ na base de dados da Biblioteca Virtual em Saúde (BVS) utilizando os descritores Acolhimento, Estratégia Saúde da Família, NASF e Gestão. As referências encontradas apoiaram a discussão dos resultados encontrados com a observação sistemática e com as entrevistas.

A observação sistemática ${ }^{10}$ foi realizada como apoio às entrevistas e permitiu identificar o envolvimento dos profissionais com o acolhimento nas unidades, assim como a utilização do acolhimento e do NASF para planejamento de açôes pela gestão. Foi utilizado para tal um roteiro pré-definido que foi aplicado em dois turnos por unidade, perfazendo um total de 20 horas de observação.

A entrevista semiestruturada ${ }^{9}$, por sua vez, foi o principal método utilizado e possibilitou identificar, através das falas dos profissionais, o conhecimento relacionado às temáticas acolhimento e NASF, assim como a importância desses como ferramenta para gestáo das unidades. Um roteiro previamente elaborado foi utilizado para conduzir as entrevistas. Entrevista-piloto com um profissional enfermeiro foi realizada para avaliar a viabilidade do instrumento.

Para análise dos resultados, foi realizada inicialmente uma leitura flutuante com base em Bardin ${ }^{11}$ de todo o material coletado no campo e nas referências. Na leitura flutuante surgem hipóteses ou questôes norteadoras, em função das teorias já conhecidas, e, assim, ela permitiu identificar nas falas dos profissionais termos relacionados ao objetivo deste artigo. Esses termos foram agrupados em tabelas que foram organizadas de acordo com três categorias analíticas: conhecimento relacionado ao acolhimento e ao NASF; contribuição do acolhimento e do NASF para o direcionamento das açôes de saúde; e importância do acolhimento e do NASF como ferramentas de apoio à gestão. Nessas tabelas, considerando o tipo de unidade e a categoria dos profissionais, procuraram-se identificar conceitos presentes nas falas dos profissionais que se relacionavam com os conceitos prescritos nas principais políticas sobre o assunto. Esses conceitos, quando agrupados, traziam em sua estruturação uma análise do cotidiano de trabalho das duas unidades, permitindo identificar o acolhimento prescrito e o acolhimento real, o envolvimento dos profissionais e o papel do acolhimento e do NASF enquanto ferramentas de apoio à gestão.

\section{Resultados e discussão}

A Tabela 1 descreve o perfil dos profissionais entrevistados, considerando a categoria profissional, o tempo de atuação nas unidades, a formação, o regime trabalhista e a carga horária.

A maioria dos entrevistados possuía mais de um ano de atuação nas UBS. Somente os gerentes das UBS, um médico, um enfermeiro e uma das nutricionistas do NASF possuíam especialização em Saúde da Família. Todos os profissionais cumpriam carga horária semanal de 40 horas.

O curso introdutório (obrigatório para atuação na estratégia saúde da família) foi realizado por todos os profissionais que atuavam na ESF e não foi considerado na resposta referente ao item formação. $\mathrm{Na} \mathrm{CF}$, os únicos profissionais estatutários eram os AVS, sendo os outros celetistas; por sua vez, no CMS existiam profissionais estatutários e celetistas nas diferentes categorias entrevistadas.

O acolhimento foi visto, através da observação e das entrevistas, como uma das principais ferramentas de gestão e de regulação do acesso das UBS, além de importante instrumento para construção de açôes de saúde mais efetivas. Mas que acolhimento foi observado nas UBS? Aquele que se concretiza no cotidiano das práticas de saúde por meio da escuta qualificada e da capacidade de pactuaçáo entre a demanda do usuário e a possibilidade de resposta do serviço. Ele se traduz em qualificação da produção de saúde, complementando-se com a responsabilização daquilo que não se pode responder de imediato, mas que é possível direcionar de maneira ética e resolutiva, com segurança de acesso ao usuário. ${ }^{12}$ 
Tabela 1. Perfil dos profissionais entrevistados.

\begin{tabular}{|c|c|c|c|c|c|c|c|c|}
\hline \multirow[t]{2}{*}{ Categoria profissional } & \multicolumn{2}{|c|}{$\begin{array}{c}\text { Tempo de atuação na } \\
\text { unidade }\end{array}$} & \multicolumn{2}{|c|}{$\begin{array}{c}\text { Formação específica em } \\
\text { saúde da família }\end{array}$} & \multicolumn{2}{|c|}{ Regime trabalhista } & \multicolumn{2}{|c|}{ Carga horária (horas } \\
\hline & $\mathrm{CF}$ & CMS & $\mathrm{CF}$ & CMS & $\mathrm{CF}$ & CMS & $\mathrm{CF}$ & $\mathrm{CMS}$ \\
\hline Gerente & $>1$ ano & $>1$ ano & Sim & $\operatorname{Sim}$ & Celetista & Celetista & 40 & 40 \\
\hline Médico & $<1$ ano & $>1$ ano & Sim & Não & Celetista & Celetista & 40 & 40 \\
\hline Enfermeiro & $>1$ ano & $>1$ ano & Sim & Não & Celetista & Estatutário & 40 & 40 \\
\hline Nutricionista do NASF & $>1$ ano & $>1$ ano & Não & Sim & Celetista & Celetista & 40 & 40 \\
\hline Agente comunitário de saúde & $>1$ ano & $>1$ ano & Não & Não & Celetista & Celetista & 40 & 40 \\
\hline Agente de vigilância em saúde & $>1$ ano & $>1$ ano & Não & Não & Estatutário & Estatutário & 40 & 40 \\
\hline Técnico de higiene dental & $>1$ ano & $>1$ ano & Não & Não & Celetista & Celetista & 40 & 40 \\
\hline Técnico de enfermagem & $>1$ ano & $<1$ ano & Não & Não & Celetista & Estatutário & 40 & 40 \\
\hline Auxiliar administrativo & $>1$ ano & $>1$ ano & Não & Não & Celetista & Celetista & 40 & 40 \\
\hline Auxiliar de serviços gerais & $>1$ ano & $<1$ ano & Não & Não & Celetista & Celetista & 40 & 40 \\
\hline Auxiliar de portaria & $>1$ ano & $>1$ ano & Não & Não & Celetista & Celetista & 40 & 40 \\
\hline
\end{tabular}

Esse acolhimento foi observado considerando o envolvimento dos profissionais com o acolhimento realizado e o entendimento da necessidade de responsabilização por buscar respostas para as necessidades de saúde encontradas. Buscou-se, assim, compreender quem era responsável por orientar e direcionar os usuários para as açóes, grupos e serviços que as unidades ofereciam. Para o atendimento das demandas, um plano de acolhimento único e protocolos clínicos orientados por linhas de cuidado complementavam as ações realizadas e auxiliavam na avaliaçáo de risco e vulnerabilidades.

Foi possível identificar, através do discurso dos profissionais, o grau de responsabilização por acolher, direcionar e utilizar de forma plena os dispositivos que as unidades possuíam e que serviam de apoio para respostas positivas às demandas dos usuários:

Eu tenho preconizado que qualquer pessoa que esteja no corredor que seja indagada alguma coisa que saiba qual é o fluxo porque até o médico quando sai para ir até o banheiro ou tomar uma água ou fazer um lanche, tomar um café, ele deve ser capaz de informar o paciente, de acolher e dar as informaçóes necessárias. (Médico)

Todos os profissionais estão na verdade preparados para dar esse tipo de informação e nós temos bastante informações nos quadros. Das datas, dos dias. Temos grupo de gestante, de tabagismo, de planejamento. Tudo isso, ou você direciona para o profissional que é capacitado para poder ver como fazer, ou você encaminha para o agente de saúde que vai dar a informação de quando ele pode vir. (Técnico de enfermagem)

Em geral são encaminhados pelos profissionais que fazem os atendimentos. Os médicos, as enfermeiras, os agentes e os técnicos. Geralmente eles encaminham, aconselham o paciente a participar. Nós fazemos divulgação com palestras, com folhetos, com panfletos, fazemos sim. (Auxiliar administrativo)

Qualquer profissional pode encaminhar para os grupos independente da profissão. Mas geralmente a gente manda para o acolhimento porque eles sabem certo os dias dos grupos. (Enfermeiro)

Utilizamos como base de discussão o conceito de tecnologia em saúde, utilizado por Merhy. O autor considera três tipos de tecnologias, classificadas em leves, que associa as relaçôes de produção de vínculo, construção da autonomia, acolhimento e gestão; as leve-duras, que seriam os saberes já estruturados, tais como a clínica médica, a clínica psicanalítica, a epidemiologia, o taylorismo e o fayolismo; e as tecnologias duras, quais sejam, as máquinas, as normas e as estruturas organizacionais. ${ }^{6}$

As entrevistas e a observação possibilitaram conhecer a inserção do Núcleo de Apoio à Saúde da Família nas duas unidades estudadas, bem como o formato das tecnologias em saúde utilizadas. Em relação ao acolhimento, este foi identificado como sendo essencialmente relacional. Quando focamos no NASF, observamos que em alguns momentos utilizou as tecnologias leves e, em outros, as tecnologias leve-duras, dando vazão às várias possibilidades de uso do trabalho em saúde. 
As UBS possuíam normas específicas e instrumentos que normatizavam o atendimento aos usuários e que, portanto, foram identificados como tecnologias duras. Essas normas e instrumentos foram os seguintes: regulamento e regimento interno, plano de acolhimento, instrumentos para atendimento específico em saúde da criança, da mulher, do homem e do idoso, bem como instrumentos específicos para atuação em sala de vacina, curativo, procedimento, visita domiciliar e atendimento em grupo.

O acolhimento, enquanto tecnologia leve possibilitava a identificação das necessidades reais do usuário; por sua vez, as tecnologias leve-duras e duras que as unidades possuíam contribuíam para resolutividade dessas necessidades.

Através das falas dos profissionais, procuramos identificar como eram utilizadas as tecnologias em saúde (serviços, procedimentos, grupos) para direcionamento e atendimento das necessidades dos usuários a partir do acolhimento. Assim, o agente comunitário de saúde foi apontado pelas entrevistas como principal responsável por acolher os usuários.

No entanto, através da observaçáo sistemática, foi possível constatar que as atividades realizadas eram sinalizadas através de informativos que ficavam visíveis nos quadros anexados nos corredores das unidades. Esses informativos divulgavam os grupos, açôes e atividades planejados pelos profissionais.

Os serviços ofertados pelas unidades estavam sistematizados através de fluxogramas que orientavam o processo de trabalho. Esses, por sua vez, permitiam atendimento nos diferentes ciclos de vida e atendiam a inúmeras necessidades de saúde dos usuários respondendo às demandas encontradas no acolhimento. $\mathrm{O}$ direcionamento e encaminhamento para esses serviços, no entanto, poderia ser realizado por qualquer profissional que tivesse conhecimento prévio das tecnologias em saúde existentes na unidade e estivesse intimamente envolvido com o processo de trabalho das unidades. A entrada de usuários nas UBS nem sempre estava condicionada a atendimento médico e de enfermagem, e os fluxos definidos orientavam os profissionais, contribuindo para escuta qualificada, estabelecendo critérios para encaminhamento para os grupos, permitindo levantamento das principais demandas de atendimento das unidades e planejamento de açóes específicas voltadas para as demandas com maior índice de procura pelos serviços. Por vezes, foi visto pronto atendimento da demanda através da utilização de outros serviços que as unidades possuíam, tais como imunização, saúde bucal, curativos, grupos de promoção em saúde e visita domiciliar.

Por sua vez, os fluxos para orientar o trabalho na APS, as tecnologias em saúde e o planejamento devem ser desenvolvidos com apoio do NASF, uma vez que ele insere outros profissionais no processo de gestáo compartilhada do cuidado e contribui para aumentar a resolutividade. ${ }^{13}$ Quanto maior o grau de envolvimento do NASF com o acolhimento, maior a possibilidade de profissionais mais resolutivos. O NASF, pensado enquanto tecnologia, pode ser visto como dispositivo de apoio para o acolhimento, uma vez que aumenta a capacidade resolutiva da Atenção Primária. Os NASFs devem buscar contribuir para a integralidade do cuidado aos usuários do SUS, principalmente por intermédio da ampliação da clínica, auxiliando no aumento da capacidade de análise e de intervençáo sobre problemas e necessidades de saúde, tanto em termos clínicos quanto sanitários. São exemplos de açôes de apoio desenvolvidas pelos profissionais dos NASF: discussão de casos, atendimento conjunto ou não, interconsulta, construção conjunta de projetos terapêuticos, educação permanente, intervençōes no território e na saúde de grupos populacionais e da coletividade, açôes intersetoriais, açôes de prevenção e promoção da saúde e discussão do processo de trabalho das equipes. ${ }^{12}$

Percebeu-se, através das falas dos profissionais, coerência relacionada às atribuiçóes dos profissionais do NASF e sua importância para aumentar a resolutividade das equipes de saúde da família:

Funciona como um capacitador. (Médico)

O principal papel do NASF é o matriciamento. Estấo inseridos em todas as atividades e nos grupos, realizam visitas domiciliares. (Gerente)

O ideal é que sempre aconteça nas reuniôes de equipe porque a gente consegue implicar um número maior de pessoas e todo mundo vai ter a mesma direçấo. E aí a partir disso a gente faz interconsulta, vai à casa da pessoa fazer visita domiciliar ou a gente pode fazer atendimento individual se for o caso.(NASF)

Já vi atender por grupos e por paciente. (Auxiliar de serviços gerais)

Eles fazem consultas conjuntas, buscam solucionar os problemas e, quando há necessidade, o profissional do NASF sai a campo. Participam das reuniôes de equipe. (Auxiliar administrativo) 
Considerando o papel do NASF descrito pela $\mathrm{PNAB}^{14}$ e pelo Caderno de Atenção Básica no 27:13

O NASF deve atuar dentro de algumas diretrizes relativas à APS, a saber: ação interdisciplinar e intersetorial; educação permanente em saúde dos profissionais e da população; desenvolvimento da noção de território; integralidade, participação social, educação popular; promoção da saúde e humanização. A organização dos processos de trabalho dos NASF, tendo sempre como foco o território sob sua responsabilidade, deve ser estruturada priorizando o atendimento compartilhado e interdisciplinar, com troca de saberes, capacitação e responsabilidades mútuas, gerando experiência para todos os profissionais envolvidos, mediante amplas metodologias, tais como estudo e discussão de casos e situações, projetos terapêuticos, orientaçôes e atendimento conjunto etc. (BRASIL, 2012, p.07)

Pode-se observar que parte dos profissionais que atuavam nas unidades entendia a atuação e a inserção do NASF. Foram apontados pelos profissionais como formas de atuação e inserção do NASF: participação em reunião de equipe, grupos e açóes, interconsultas e visitas domiciliares. $\mathrm{O}$ atendimento individual também foi considerando como importante face às demandas específicas. O NASF foi considerado ainda como mecanismo para educação permanente e capacitação dos profissionais e como suporte para atendimento em grupo e para realização de açôes na unidade e no território.

No que concerne ao atendimento dispensado aos usuários com problemas relacionados à saúde mental, o NASF foi identificado como extremamente necessário. No entanto, foi possível observar dificuldade de entendimento relacionado à atuação do NASF por parte dos profissionais que atuam no apoio, tais como auxiliares de portaria, agente de vigilância em saúde e auxiliares de serviços gerais. Por vezes, não sabiam da existência do NASF, ou sabiam da existência, mas não entendiam de que forma o NASF atuava:

Eu também não tenho conhecimento sobre o NASF. (Agente de vigilância em saúde)

Não sei. Sei que tem um educador físico que faz caminhada às vezes com idosos e que tem nutricionista e psicólogo aqui. Mais não é todo dia e não sei como atendem. (Auxiliar de portaria)

Não sei dizer. Sei que tem aqui porque ouvi falar. Vejo os agentes conversando. (Auxiliar de serviços gerais)

As tecnologias em saúde existentes na unidade (leves, leve-duras e duras) e o NASF contribuíam significativamente com o acolhimento, uma vez que aumentavam a resolutividade no atendimento dispensado aos usuários nas unidades. Todavia, observou-se a necessidade de elaborar protocolos e fluxogramas que direcionem e ordenem o cuidado a partir do acolhimento e que impliquem o profissional, responsabilizando-o com o acolhimento a partir do primeiro contato com o usuário. Todos os profissionais que atuam nas unidades devem conhecer o processo de trabalho das equipes e do NASF, assim como dos serviços que as unidades oferecem e o lugar que a unidade ocupa na rede de serviços em saúde. Percebeu-se frágil discurso sobre estas questóes por parte dos gerentes e dificuldade de entendimento da importância do acolhimento e do NASF enquanto políticas capazes de contribuir com a gestão.

\section{Conclusões}

A formação clínica como produtora do cuidado em saúde ${ }^{15}$ agrega uma intencionalidade resolutiva, evocando a conformação de um sistema de saúde que integre os diversos pontos de atenção à saúde, em rede; e uma intencionalidade mobilizadora de subjetividades, que agrega a reconstrução das relaçôes entre os sujeitos. O acolhimento apresentou-se nas UBS ora como elemento essencial para fortalecimento da construçáo de uma rede de atençáo à saúde que possui como porta de entrada principal a Atenção Primária, ora como elemento capaz de contribuir para regulação do acesso. Mostrou-se ainda, potente para reconstruçáo de práticas a partir das necessidades de saúde dos sujeitos. A aposta é em um trabalho em equipe onde o foco esteja na produção do cuidado em saúde, onde as relaçóes de poder, de saber e a subjetividade, consideradas essenciais para o encontro entre os sujeitos, possa ser dispositivo mobilizador.

Reafirmamos o acolhimento como essencial para a organização da porta de entrada nas Unidades Básicas de Saúde, já que possibilita escuta qualificada, atendimento das diferentes demandas e utilização dos serviços disponibilizados pela rede de atenção à saúde, compreendendo-o como espaço de qualificação do SUS, que vem cada vez mais assumindo o desafio de garantir avaliação e monitoramento de suas políticas públicas e práticas ${ }^{16}$. 
Nesta lógica, valorizamos o NASF como dispositivo de apoio ao acolhimento, já que permite intervençôes amplas onde os profissionais encontram fragilidades no manejo de problemas de saúde e na utilização de fluxos de referência em uma rede de atenção fragmentada.

No entanto, inferimos caminhos sublinhando o olhar para o NASF que desejamos: mais implicado com o processo de desenvolvimento de competências da equipe mínima na Estratégia Saúde da Família e tendo maior envolvimento e participação nas açôes de promoção da saúde e apoio às atividades planejadas pela gerência das Unidades Básicas de Saúde.

\section{Referências}

1. Friedrich DBC, Pierantoni CR. O trabalho das equipes da saúde família: um olhar sobre as dimensões organizativa do processo produtivo, político-ideológica e econômica em Juiz de Fora. Physis. 2006 Jan [acesso em 2013 Sep 07];16(1):83-97. http://dx.doi.org/10.1590/S0103-73312006000100006

2. Malik AM, Braga Junior D, Braga DG, Silva JC, Amorin JCM. Gestão de Recursos Humanos. São Paulo: Faculdade de Saúde Pública da Universidade de São Paulo; 1998. (Série Saúde \& Cidadania, vol. 9)

3. Campos GWS. Equipes de referência e apoio especializado matricial: um ensaio sobre a reorganização do trabalho em saúde. Ciênc Saúde Coletiva. 1999 May [acesso em 2013 Aug 03]; 4(2):393-403. http://dx.doi.org/10.1590/S1413-81231999000200013

4. Campos GWS. Subjetividade e administração de pessoal: considerações sobre modos de gerenciar o trabalho em equipes de saúde. In: Merhy EE, Onocko R, editors. Agir em saúde: um desafio para o público São Paulo: Hucitec; 1997. p. 229-66.

5. Merhy EE, Chakkour M, Stéfano E, Santos CM, Rodrigues RA, Oliveira PCP. Em busca de ferramentas analisadoras das tecnologias em saúde: A informação e o dia-a-dia de um serviço, interrogando e gerindo o trabalho em saúde. In: Merhy EE, Onocko R, editors. Agirem saúde: Um desafio para o público. São Paulo: Hucitec; 1997. p.113-50.

6. Merhy EE. O Ato de Cuidar: A Alma dos Serviços de Saúde. In: Ministério da Saúde. Secretaria de Gestão do Trabalho e da Educação na Saúde, Departamento de Gestão da Educação na Saúde. Ver - SUS Brasil: Cadernos de textos. Brasília: MS; 2004. p.108-137.

7. Santana ML. Demanda espontânea e planejamento estratégico no PSF de Pindamonhangaba. Rev Bras Med Fam Comunidade. Florianópolis, 2011 Abr-Jun; 6(19): 133-41. http://dx.doi.org/10.5712/rbmfc6(19)163

8. Secretaria Municipal de Saúde do Rio de Janeiro. Carteira de serviços - relação de serviços prestados na Atenção Primária. Rio de Janeiro [acesso em 2013 Nov 09]. Disponível em: http://subpav.org/index.php?p=cart

9. Minayo MC. Desafio do Conhecimento: Pesquisa qualitativa em saúde. 10ª ed. São Paulo: Hucitec; 2007.

10. Becker HSM. Métodos de pesquisa em ciências sociais. São Paulo: Hucitec; 1997.

11. Bardin L. Análise de conteúdo. Coimbra (PT): Lisboa; 1977.

12. Ministério da Saúde (BR), Secretaria de Atenção à Saúde, Departamento de Atenção Básica. Acolhimento à demanda espontânea: queixas mais comuns na Atenção Básica. Cadernos de Atenção Básica. Brasília: MS; 2012.

13. Ministério da Saúde (BR), Secretaria de Atenção à Saúde, Departamento de Atenção Básica. Diretrizes do NASF (Núcleo de Apoio à Saúde da Família). Cadernos de Atenção Básica. Brasília: MS; 2009.

14. Ministério da Saúde (BR), Secretaria de Atenção à Saúde, Departamento de Atenção Básica. Política Nacional de Atenção Básica. Brasília: MS; 2012.

15. Vieira AN, Silveira LC, Franco TB. A Formação clínica e a produção do cuidado em saúde e na enfermagem. Trab Educ Saúde. 2011 Mar/Jun [acesso em 2014 Nov 08]; 9(1):9-24. http://dx.doi.org/10.1590/S1981-77462011000100002

16. Deslandes DF, Mendes CHF, Luz ES. Análise de desempenho de sistema de indicadores para o enfrentamento da violência intrafamiliar e exploração sexual de crianças e adolescentes. Ciênc Saúde Coletiva. 2014 Mar [acesso em 2014 Nov 09]; 19(3):865-874. http://dx.doi.org/10.1590/1413-81232014193.06012013 